\title{
Multiple functions of the first EGF domain in matrilin-3: Secretion and endoplasmic reticulum stress
}

\author{
YI-CHUN WANG ${ }^{1,2}$, JING-SHI LIU ${ }^{2}$, JUN-YI CHEN ${ }^{2}$, SHENG-QI WU ${ }^{3}$, GUI-RONG WANG ${ }^{4}$, \\ JING NIE $^{2}$, SHU-KUN ZHANG ${ }^{5}$, QU-LIAN GUO ${ }^{1}$ and JUN-MING LUO ${ }^{5}$ \\ ${ }^{1}$ Department of Anesthesiology, Xiangya Hospital, Central South University, Changsha, Hunan 410008; \\ ${ }^{2}$ Department of Critical Care Medicine and ${ }^{3}$ Central Laboratory, The Affiliated Cancer Hospital \\ of Xiangya Medical School, Central South University, Changsha, Hunan 410013, P.R. China; \\ ${ }^{4}$ Department of Surgery, SUNY Upstate Medical University College of Medicine, Syracuse, NY 13210, USA; \\ ${ }^{5}$ Department of Pathology, Qinghai People's Provincial Hospital, Xining, Qinghai 810007, P.R. China
}

Received April 2, 2015; Accepted October 2, 2015

DOI: $10.3892 / \mathrm{ijmm} .2015 .2377$

\begin{abstract}
Mutations in matrilin-3 are associated with common skeletal diseases, such as hand osteoarthritis (HOA), as well as rare chondrodysplasias, such as multiple epiphyseal dysplasia (MED) and spondyloepimetaphyseal dysplasia (SEMD). In the present study, we constructed the mutations R116W [at the von Willebrand factor, type A (vWFA) domain], T298M [at the first epidermal growth factor (EGF) domain] and C299S (at the first EGF domain), according to the mouse sequence, which are associated with human MED, HOA and SEMD, respectively, by overlap extension PCR and inserted them into an expression vector (pcDNA3.1/v5-His). We transfected these contructs into the COS-1 or MCT cells, and the results revealed that the HOA-related matrilin-3 mutation (T298M) leads to a high expression level of growth arrest DNA damage-inducible gene 153 (GADD153, also known as CHOP; an endoplasmic reticulum stress marker), as shown by western blot analysis and does not significantly affect protein secretion, as shown by immunofluorescence staining; however, osteochondroplasia,
\end{abstract}

Correspondence to: Professor Qu-Lian Guo, Department of Anesthesiology, Xiangya Hospital, Central South University, 87 Xiangya Road, Changsha, Hunan 410008, P.R. China

E-mail: qulianguo@hotmail.com

Professor Jun-Ming Luo, Department of Pathology, Qinghai People's Provincial Hospital, 2 Gonghe Road, Xining, Qinghai 810007, P.R. China

E-mail: jluo099@163.com

Abbreviations: PAGE, polyacrylamide gel electrophoresis; RT-PCR, reverse transcription-polymerase chain reaction; MED, multiple epiphyseal dysplasia; OA, osteoarthritis; HOA, hand osteoarthritis; SEMD, spondyloepimetaphyseal dysplasia; ER, endoplasmic reticulum; COMP, cartilage oligomeric matrix protein; EGF, epidermal growth factor

Key words: matrilin-3, point mutation, chondrodysplasia, hand osteoarthritis, secretion, endoplasmic reticulum stress i.e., MED-related (R116W) and SEMD-related (C299S) mutations lead to both high levels of GADD153 expression and protein trafficking into the cytoplasm and form multiple vacuoles in cells, which in turn leads to insufficient protein secretion.

\section{Introduction}

In cartilage, the extracellular matrix (ECM) occupies $95 \%$ of the space which plays a dominant role in chondrocyte functions. ECM is composed of a large number of different molecules, which mediate cell-matrix and matrix-matrix communications, thereby determining the histoarchitecture specific to each organ, and provide cells with crucial information on migration, adhesion and differentiation (1-4). There are three classes of ECM molecules, which include filamentous collagen molecules, proteoglycans and non-filamentous collagen molecules (1-4). Matrilins are a family of four filamentous-forming adapter oligomeric extracellular proteins that are involved in the development and homeostasis of cartilage and bone (1,5-7). They can form homo- and hetero-oligomers through the assembly of C-terminal coiled-coil structures (1,5-7). As regards their distribution, it has been demonstrated that matrilin-1 and -3 are restricted to cartilage and bone, whereas matrilin- 2 and -4 are present in a broader range of tissues $(1,2,8,9)$. Matrilins are thought to play a role in the ECM by acting as bridges which connect matrix components and the cell membrane in the cartilage to form macromolecular networks $(10,11)$. In cartilage, matrilins interact with type II and type IV collagen, and aggrecan, as well as the cartilage oligomer matrix protein (COMP) $(1,10,11)$. Matrilins consist of one or two von Willebrand factor, type A (vWFA) domains, a varying number of epidermal growth factor (EGF) repeats, and a C-terminal coiled-coil domain (1-3). The polypeptide of matrilin-3 predicted from the nucleotide sequence of this clone shared $83 \%$ identity with matrilin-3 from mouse and $61 \%$ with that from chicken $(5,12,13)$. It was composed of 486 amino acid residues that were arranged in 7 domains: a signal peptide, a vWFA domain, four EGF repeats and an $\alpha$-helical region. The gene for human matrilin- 3 was assigned to chromosome region 2 . The corresponding mRNA of $2.8 \mathrm{~kb}$ 
has been found to be expressed in every type of cartilage investigated thus far $(5,12,13)$.

The vWFA domain is found in various collagenous and non-collagenous ECM proteins, and is composed of a central $\beta$-sheet core flanked by $\alpha$-helices $(5,10,11)$. In humans, at least nine missense point mutations in the matrilin- 3 gene that affect the vWFA domain (typically the $\beta$-sheets) have been found in patients with multiple epiphyseal dysplasia (MED), which is characterized by delayed and irregular ossification of the epiphyses and early onset osteoarthritis (OA) (14-20). Mutations of matrilin-3 have also been reported in other osteochondrodysplasias, including bilateral hereditary microepiphyseal dysplasia, which is an MED-like disorder characterized by small epiphyses in the hip and knee joints (14-20).

EGF domain-containing molecules have also been identified ubiquitously in the ECM, cell membrane and nucleus and similar to vWFA domain-containing molecules, they participate in cell adhesion, protein-protein interactions and the formation of multiprotein complexes $(21,22)$. Mutations in the first EGF domain of matrilin-3 have also been reported in osteochondrodysplasias, such as spondyloepimetaphyseal dysplasia (SEMD), which includes a number of conditions associated with vertebral, epiphyseal and metaphyseal anomalies (23) and idiopathic hand osteoarthritis (HOA) (24).

Matrilin-3 has also been reported to be upregulated in cartilage from patients with $\mathrm{OA}$, and a strong correlation between enhanced matrilin-3 gene and protein expression and the extent of tissue damage has been noted $(5,12,25)$.Matrilin-3 is also produced in vitro by primary chondrocytes isolated from articular cartilage. However, dedifferentiated chondrocytes of the third passage do not express matrilin-3 at all (12). Therefore, it has been suggested that matrilin-3 serves as a marker for the differentiation state of chondrocytes $(5,12,13,25)$. These findings suggest that the tight regulation of matrilin-3 expression is essential for the maintenance of the cartilage ECM microenvironment.

In mice, matrilin-3 is expressed exclusively in the developing cartilage of the skeletal system, with its expression first being detected on embryonic day 12.5 and remains unaltered from embryonic day 15.5 until birth. In newborn mice, matrilin-3 is widely expressed in the cartilage of the developing bones of the eyes, nasal cavity, ribs, long bones, sternum and trachea, although its expression is more restricted by 6 weeks of age $(5,12,13)$. Although it has been shown that matrilin-1 and -3 can form hetero-oligomers and are often co-localized in tissue, clear differences in their spatial distribution have been shown by double immunolabeling $(5,12,13)$. In addition, although matrilin-1 expression continues to be present in tissues that remain cartilaginous throughout life (such as in costal cartilage and in the nasal septum), matrilin-3 expression ceases to be present after birth in these tissues.

\section{Materials and methods}

Extraction of RNA from animal tissues, reverse transcriptionpolymerase chain reaction (RT-PCR) and preparation of wild-type and point mutation of matrilin-3 gene constructs. A total of 3 newborn C57BL/6 mice (Charles River Laboratories, Wilmington, MA, USA) were sacrificed by $\mathrm{CO}_{2}$ asphyxiation and the rib cages were harvested. All procedures were approved by the Ethics Committee of Qinghai Provincial People's Hospital. Tissue was homogenized, and total RNA was isolated using an RNeasy kit (Qiagen, Germantown, MD, USA). First-strand cDNA was synthesized using a SuperScript II kit (Invitrogen, Grand Island, NY, USA) according to the manufacturer's instructions. Wild-type matrilin-3 cDNA was cloned from the newborn C57BL/6 mouse rib library. Specific primers (Table I) introduced a 5'-terminal BamH1 and a 3'-terminal HindIII restriction enzyme site into the expression vector, pcDNA3.1/v5-His (Invitrogen). Point mutations of R116W, T298M and C299S, according to the mouse sequence, associated with MED, HOA and SEMD, respectively, were linked by overlap extension PCR with the described primers (Fig. 1 and Table I) from the wild-type matrilin-3 plasmid. Recombinant sequences were confirmed by DNA sequencing.

Transfection of plasmid DNA. Plasmids containing wild-type, MED, SEMD, HOA and both SEMD plus HOA-point mutations of matrilin-3 were transfected into the COS-1 or MCT (chondrocytes) cells (kind gift from Dr Lei Wei, Brown University, Rhode Island Hospital, Providence, RI, USA) using Lipofectamine 2000 (Invitrogen Life Technologies, Carlsbad, CA, USA) according to the manufacturer's instructions. Briefly, the COS-1 or MCT cells were trypsinized and counted. Each 60 -mm plate was seeded with $6 \times 10^{5}$ cells overnight until the cells reached $70 \%$ confluence in Dulbecco's modified Eagle's medium (DMEM) supplemented with $10 \%$ fetal bovine serum (FBS; Invitrogen Life Technologies). On the following day, the cells were rinsed with DMEM 3 times, and then transfected with the plasmid/Lipofectamine $2000 \mathrm{mix}$, as previously described $(5,13)$. We also created a group of untransfected cells (not transfected with any plasmids) and a group of mock-transfected cells (transfected with pcDNA3.1 empty vector). The COS-1 cell-conditioned medium was collected for western blot analysis on days 1 to 5, and the MCT cell-conditioned medium was collected for western blot analysis on days 1 to 3 .

Western blot analysis. Conditioned medium $(5 \mu \mathrm{l})$ or cell lysates $(20 \mu \mathrm{g})$ was mixed with $5 \mu \mathrm{l} 2 \mathrm{X}$ SDS reducing gel loading buffer containing 5\% $\beta$-mercaptoethanol. After being boiled for $10 \mathrm{~min}$, the samples were loaded onto 4-15\% gradient gels (Bio-Rad Laboratories, Hercules, CA, USA). Following electrophoresis, the proteins were transferred onto immobilonpolyvinylidene difluoride membranes (Merck Millipore,Billerica, MA, USA). The blots were blocked with 5\% non-fat milk (BioRad Laboratories). Monoclonal antibody against V5 epitope tag (Invitrogen; dilution 1:5,000) for conditioned medium, rabbit polyclonal anti-growth arrest DNA damage-inducible gene 153 (GADD153) antibody (Santa Cruz Biotechnology, Santa Cruz, CA, USA; SC-575, dilution 1:200) and monoclonal anti- $\beta$ actin antibody (Sigma, St. Louis, MO, USA; A5441, dilution 1:5,000) were used as the primary antibodies. The secondary antibodies were horseradish peroxidase-conjugated goat antimouse IgG $(\mathrm{H}+\mathrm{L})$ (Bio-Rad Laboratories; dilution 1:5,000) or horseradish peroxidase-conjugated goat anti-rabbit IgG $(\mathrm{H}+\mathrm{L})$ (Bio-Rad Laboratories; dilution 1:5,000). The visualization of immunoreactive proteins was carried out using ECL western blotting detection reagents (Amersham Pharmacia Biotech Inc., Piscataway, NJ, USA) followed by exposure of the membranes to Kodak X-OMAT AR film. The molecular weights of the immu- 
Table I. Primers used in the present study.

\begin{tabular}{lll}
\hline Primers & \multicolumn{1}{c}{ Primer sequences $\left(5^{\prime} \rightarrow 3^{\prime}\right)$} & \multicolumn{1}{c}{ PCR purpose } \\
\hline 1 & TAA TAC GAC CTA TAG GG & T7, amplifying inserts from pCDNA3.1 \\
2 & CTA GAA GGC AAC AGT CGA GG & BGH, amplifying inserts from pCDNA3.1 \\
3 & CCCAAGCTTATGTTGCTCTCAGCCCCCTTA & Clone matrilin-3 with HindII \\
4 & CCGGATCCTTAACGATGTACTTGTCCAT & Clone matrilin-3 with Bam 1 \\
5 & ATGGGAAAATGTGTTCAGCCATTGATA & T298M mutation \\
6 & CAATGGCTGAACACATTTTCCCATCAGCGT & T298M mutation \\
7 & GGAAAACGTCTTCAGCCATTGATAAGT & C299S mutation \\
8 & CAATGGCTGAAGACGTTTTCCATCAG & C299S mutation \\
9 & ATCTGCGTTAGAGCCACAACAAGCAGT & R116W mutation \\
10 & AAAGAACAACTTGGGTTCATT & R116W mutation \\
\hline
\end{tabular}

1. Hand osteoarthritis

OA-MATN3

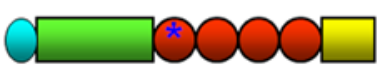

2. Spondyloepimetaphyseal dysplasia

SEMD-MATN3
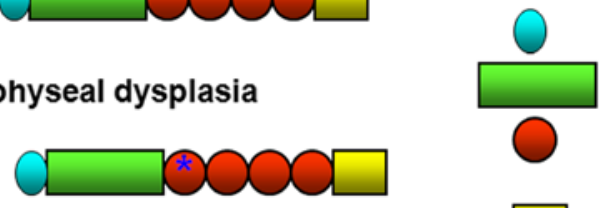

Signal peptide

vWFA

EGF

$\alpha$-Helix

3. Multiple epiphyseal dysplasia and $O A$

MED-MATN3

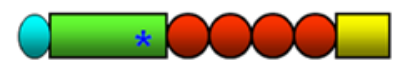

* Point mutation

Figure 1. Schematic representation of the domain structure of recombinant matrilin-3 variants and point mutations. We constructed 3 plasmids carrying the following point mutations: the multiple epiphyseal dysplasia (MED) (R116W) mutation located at the von Willebrand factor, type A (vWFA) domain, the hand osteoarthritis (HOA) (T298M), and spondyloepimetaphyseal dysplasia (SEMD) (C299S) mutations located at first EGF-like domain.

noreactive proteins were determined with two sets of protein markers, as previously described $(1,5,13)$.

Immunofluorescence staining. The COS-1 and MCT cells were seeded onto 8-well chamber slides, and $1 \mu$ g wild-type matrilin-3 or the MED-, SEMD- and HOA-associated mutations, or SEMD plus HOA-mutant matrilin-3 cDNA were transfected into the cells in each well using Lipofectamine 2000 (Invitrogen). Three days following transfection, the cells in monolayer were fixed with $70 \%$ ethanol and $50 \mathrm{mM}$ glycine for $1 \mathrm{~h}$. Immunofluorescence staining was performed by incubating the cells with anti-V5 primary antibody (Invitrogen) at 1:1,000 for $2 \mathrm{~h}$, followed by incubation with donkey anti-mouse rhodamine secondary antibody (The Jackson Laboratory, Bar Harbor, ME, USA) at 1:200 dilutions in the presence of Hoechst staining solution (Sigma). The slides were mounted with coverslips in Gel/Mount.

Electron microscopy. After the MCT cells were seeded onto 60 -mm plates, $3 \mu \mathrm{g}$ wild-type matrilin-3 or SEMD, or SEMD plus HOA-mutant matrilin-3 cDNA were transfected into the cells using Lipofectamine 2000 (Invitrogen). Three days following transfection, monolayer cells were trypsinized and centrifuged. The cell pellets were washed in DMEM 3 times and then fixed in $1.5 \%$ glutaraldehyde $1.5 \%$ paraformaldehyde containing $0.05 \%$ tannic acid, followed by $1 \% \mathrm{OsO}_{4}$, then rinsed, dehydrated and embedded in Spurrs epoxy. Ultrathin sections were cut at 60-80 nm, mounted onto formvar-coated $1 \times 2-\mathrm{mm}$ slot grids, stained with uranyl acetate and lead citrate, and imaged at $120 \mathrm{kV}$ using a Hitachi H800 transmission electron microscope.

Measurement of expression levels of matrilin-3 in knee joints of Dunkin-Hartley guinea pigs by immunohistochemistry. Six 9-month-old Dunkin-Hartley guinea pigs (Charles River Laboratories) were anesthetized, and the right knee joints were harvested and decalcified with 10\% EDTA solution. All procedures were approved by the Ethics Committee of Qinghai Provincial People's Hospital. These were routinely processed and embedded in paraffin. Longitudinal serial sections of bone at thicknesses of $5 \mu \mathrm{m}$ were prepared and collected on commercially available, positively charged glass slides (Superfrost Plus; Fisher Scientific, Shanghai, China). The sections were dried on a hot plate to increase adherence to the slides. Immunohistochemistry was carried out using the avidin-biotin complex (ABC) method (Vector Laboratories, Shanghai, China). Representative sections were de-paraffined and re-hydrated through conventional methods. Endogenous peroxidase was blocked by treating the sections with $3 \%$ hydrogen peroxide in methanol for $30 \mathrm{~min}$. The sections were then digested with bovine testicular hyaluronidase, as previously described $(14,15)$ $\left(4,000 \mathrm{U} / \mathrm{ml}\right.$ in PBS; Sigma) for $30 \mathrm{~min}$ at $37^{\circ} \mathrm{C}$. Non-specific 
A

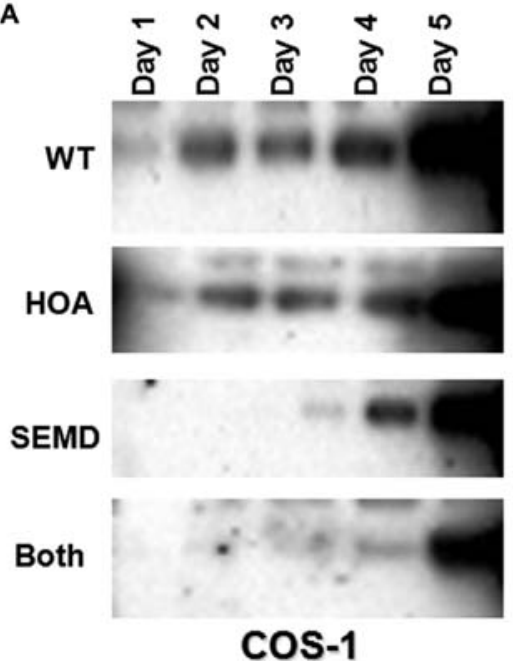

B

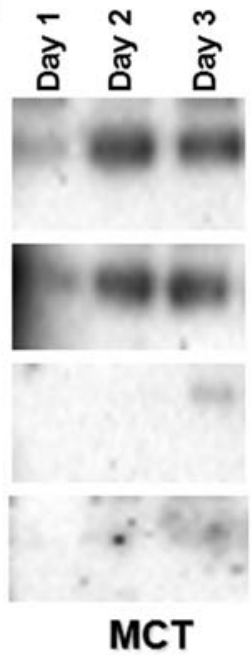

Figure 2. Secretion of matrilin-3. Western blot analysis of recombinant matrilin3-conditioned medium of (A) COS-1 cells and (B) MCT cells transfected with the plasmids carrying the different point mutations [wild-type matrilin-3 (WT) or the MED-, SEMD- and HOA-associated mutations, or SEMD plus HOA-mutan matrilin-3 cDNA (Both)]. Matrilin-3 secretion into the conditioned medium was measured on days 1 to 5 for the COS-1 cells, and on days 1 to 3 for the MCT cells by western blot analysis as described in the Materials and methods. In both the COS- 1 cells and MCT chondrocytes, the quantity and speed of secretion in the cells transfected with the SEMD-associated mutation (C299S), or with the SEMD-associated mutation (C299S) plus the HOA-mutant (T298M) of matrilin-3 were diminished in comparison to the cells transfected with wildtype or with the HAO-associated mutation (T298M) alone.

protein binding was blocked by incubation with $10 \%$ normal goat serum. The sections were incubated in a polyclonal rabbit antibody against our matrilin-3 antibody $(1: 200)$ at $4{ }^{\circ} \mathrm{C}$ overnight. Thereafter, the sections were treated sequentially with biotinylated goat anti-rabbit IgG (Vectastain Elite; Vector Laboratories) and avidin-enzyme complex (Vectastain Elite; Vector Laboratories). These treatments were followed by standardized development in 3,3'-diaminobenzidine (DAB; Zymed Laboratories Inc., South San Francisco, CA, USA). The sections were counterstained with modified Harris hematoxylin (Fisher Scientific). In order to investigate the specificity of our peptide antibody, pre-immunized serum from the same rabbit and $0.01 \mathrm{M}$ PBS service as a negative control.

\section{Results}

Osteochondrodysplasia (C299S)-related matrilin-3 point mutations lead to secretion insufficiency and protein trafficking into the cytoplasm of chondrocytes. Proteins are basic functional units of the body. Matrilin-3 is a non-collagenous extracellular protein. The secretion of matrilin-3 is dependent on primary structure and folding $(10,11,15)$. In the present study, the secretion of matrilin-3 was determined by measuring the amount of protein expressed in the conditioned medium of the cells at different time points. From days 1-5, we transfected the cells (with wild-type matrilin-3 or the MED-, SEMD- and HOA-associated mutations, or SEMD plus HOA-mutant matrilin-3 cDNA) and conditioned medium from the cells was then collected on days 1-5 for the COS-1 cells and on days 1-3 for the MCT cells. The amount of protein was determined by western blot analysis with V5 tag antibody. We found that the transfection of the
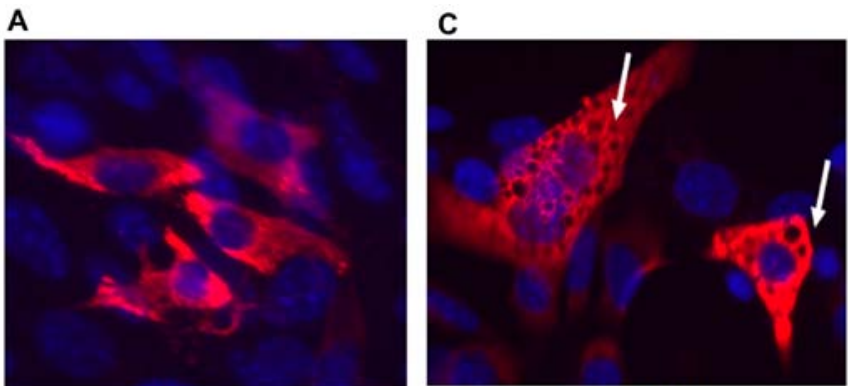

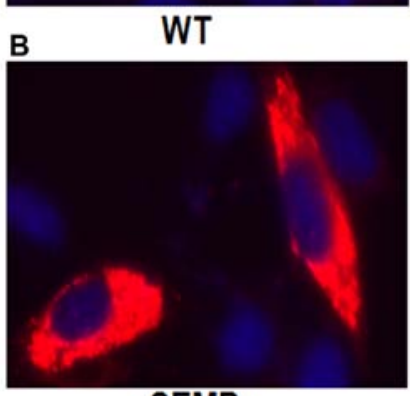

SEMD

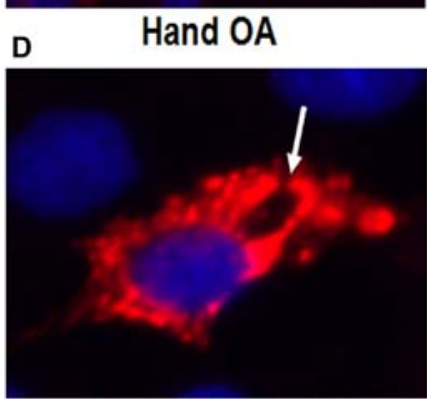

Hand OA + SEMD
Figure 3. Immunocytochemical staining for the analysis of cells transfected with the plasmids carrying matrilin-3 mutations. COS-1 cells were transfected with the plasmids carrying the different point mutations [wild-type matrilin-3 (WT) or the MED (R116W)-, SEMD (C299S)- and HOA (T298M)associated mutations, or SEMD plus HOA-mutant matrilin-3 cDNA (Both)]. Three days post-transfection, immunocytochemical analysis was performed with an antibody against the V5 tag of recombinant matrilin-3. Matrilin-3positive signals are indicated by rhodamine (red) fluorescence, whereas the cell nucleus is indicated by Hoechst dye (blue). An expanded cytoplasm was noted in the cells transfected with the SEMD-associated mutation or with SEMD plus HOA-mutant matrilin-3 cDNA, but not in the cells transfected wiht wild-type matrilin-3 or only with the HAO-associated mutation. Arrows indicate the presence of multiple vacuoles in the cells. Scale bar, $5 \mu \mathrm{m}$.

COS-1 cells with the SEMD-associated mutation or SEMD plus HOA-mutant matrilin-3 cDNA (Both) decreased the amount of protein being secreted. However, transfection with wild-type matrilin-3 (WT) or HOA-associated mutation did not prevent or decrease protein secretion into the cell medium (Fig. 2A). We also transfected the same plasmids into the MCT (chondrocytes) cells, and collected the medium on days 1 to 3 . We found that transfection of the MCT cells with SEMD-associated mutation or SEMD plus HOA-mutant matrilin-3 cDNA decreased protein secretion; however, transfection with wild-type matrilin-3 (WT) or HOA-associated mutation did not prevent protein secretion (Fig. 2B). This result is in line with that obtained with the COS-1 cells. In order to determine the morphological alterations following transfection with the matrilin-3 plasmids with the different point mutations, immunofluorescence staining was carried out. We found that there were numerous vesicles that contained the SEMD-associated mutation and the SEMD plus HOA-mutant matrilin-3, whereas by contrast, there were only a few vesicles in the cells transfected with the wild-type (WT) or HOA-associated mutation (Fig. 3). The cytoplasm of the cells transfected with mutant matrilin-3 was greatly enlarged, with multiple vacuoles. Thus, a point mutation in the cells transfected with the SEMD-associated mutation or SEMD plus HOA-mutant matrilin-3 cDNA led to a deficiency in matrilin-3 secretion, intracellular retention of the mutant protein, and an altered cytoplasm. 


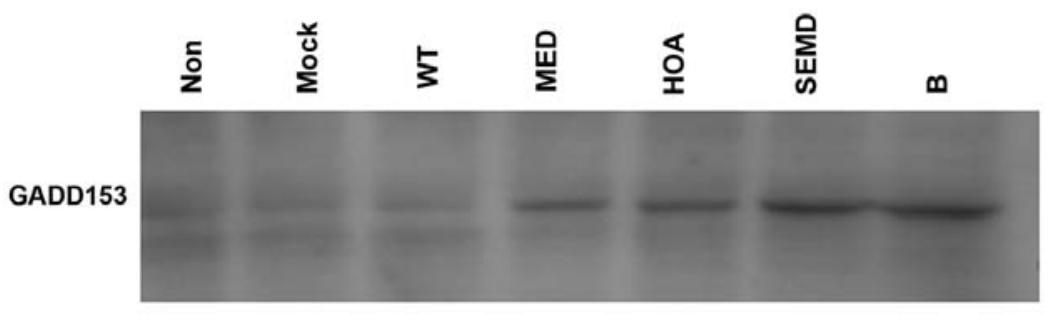

$\beta$-actin

Figure 4. GADD153 expression levels in cells transfected with plasmids carrying matrilin-3 point mutations. Western blot analysis of GADD153 expression in MCT cells transfected with the plasmids carrying the different point mutations [wild-type matrilin-3 (WT) or the MED (R116W)-, SEMD (C299S)- and HOA (T298M)-associated mutations, or SEMD plus HOA-mutant matrilin-3 cDNA (B)]. Three days post-transfection, $20 \mu \mathrm{g}$ protein from MCT cell lysates was separated on a 4-15\% SDS-PAGE gel under reducing conditions, blotted to a membrane, and incubated with antiserum against the GADD153 antisera. Bound antibodies were detected with a peroxidase-coupled secondary antibody and a chemiluminescence detection kit. There were high levels of GADD153 expression in MCT chondrocytes transfected with multiple epiphyseal dysplasia (MED), hand osteoarthritis (HOA), spondyloepimetaphyseal (SEMD) and both SEMD plus HOA-transfected cells, but low levels in the untransfected (Non), mock-transfected and wild-type matrilin-3-transfected cells.

Transfection with mutant matrilin-3 leads to endoplasmic reticulum (ER) stress. To determine the association between the first EGF-like domain of matrilin-3 and secretion insufficiencies, protein trafficking into the cytoplasm and ER stress, the expession levels of GADD153 (a key molecule in the ER stress pathway) were measured by western blot analysis. The results revealed that there was a high level of GADD153 expression in the cells transfected with the plasmids carrying all of the matrilin-3 point mutations (MED-, HOA- and SEMDassoicated mutations or SEMD plus HOA-mutant matrilin-3 cDNA); however, the expression level of GADD153 was very low in the untransfected, mock-transfected and wild-type matrilin 3-transfected cells (Fig. 4). It is important to note that there was a high level of GADD153 expression in the cells transfected with the HOA-assoicated mutation, which was not indicative of secretion insufficiencies or protein trafficking into the cytoplasm, as shown by our above-mentioned experiments (Figs. 2 and 3). This result indicate although there are some inconsistencies, a higher level of GADD153 expression may be an earlier marker for bone degenerative diseases.

Ultrastructural alterations in cells transfected with the plasmids carrying matrilin-3 point mutations. In order to examine the morphology of the COS-1 cells transfected with the plasmids carrying matrilin-3 point mutations, an electron microscope was employed. There was an abnormal cytoplasm in the cells transtected with the MED- and SEMD-associated mutations. The ultrastructural abnormalities included an expanded ER and numerous vesicles. However, the cells transfected with the HOA-associated mutation did not exhibit these abnormalities (Fig. 5).

High levels of matrilin-3 expression in developmental model of OA using Dunkin-Hartley guinea pigs. OA is one of the most common and disabling diseases affecting the elderly, and almost $80 \%$ of individuals of the age of 75 are affected $(26,27)$. OA develops due to wear and tear, combined with the loss of articular cartilage. It has been suggested that the degeneration of articular cartilage and insufficient self-repair are the primary causes of OA (25-27). In the present study, a developmental animal model of OA with Dunkin-Hartley guinea pigs was used to investigate matrilin-3 expression. The matrilin-3 expression levels were measured in the knee joints of the guinea pigs by immunohistochemistry. We found that matrilin-3 was strongly expressed in the articular cartilage, including the surface area, and proliferating and hypertrophic areas (Fig. 6).

\section{Discussion}

Matrilin-3 is the least complex member of the matrilin family, and it consists of only one vWFA domain, four EGF domains, and a C-terminal coiled-coil domain. The vWFA domain is one of the most widely distributed domains involved in cell adhesion and the formation of multiprotein complexes $(10,11)$. The property of the vWFA domain in cell adhesion and protein-protein interaction is mediated, in many cases, by the metal-ion dependent adhesion site (MIDAS) located within the domain $(5,13)$. Mutations at the vWFA domain have been reported to be related to MED. MED is an osteochondrodysplasia, primarily characterized by delayed and irregular ossification of the epiphyses and early-onset OA (12). Several different recessive mutations in the exon encoding the vWFA domain of matrilin-3 cause the EDM5 form of MED $(14,16)$. These point mutations in the vWFA domain result in single amino acid changes of T120M, R121W, E134K, I192N, V194D, A219D or E252K. Notably, all of these MED-causing mutations are located in the $\beta$ strands in the center of the vWFA domain, which are important for the folding of the protein structure (17). Subsequent genetic analysis has indicated that the $\mathrm{R} 121 \mathrm{~W}$ mutation is recurrent in multiple families with common or different ancestries (17). In the present study, we demonstrated that a single point mutation in the vWFA domain of mouse matrilin-3 (R116W), equivalent to the MED mutation (R121W) in human matrilin-3, leads to a deficiency in matrilin secretion and in the in vitro retention of matrilin-3 within the ER of primary chondrocytes, which is consistent with the findings of previous studies $(14,28)$. This point mutation also leads to a high level of GADD153 expression, which is a key marker of the ER stress pathway (29-31).

In addition, we cloned the point mutations at the first EGF domain which are associated with HOA (T298M) and 


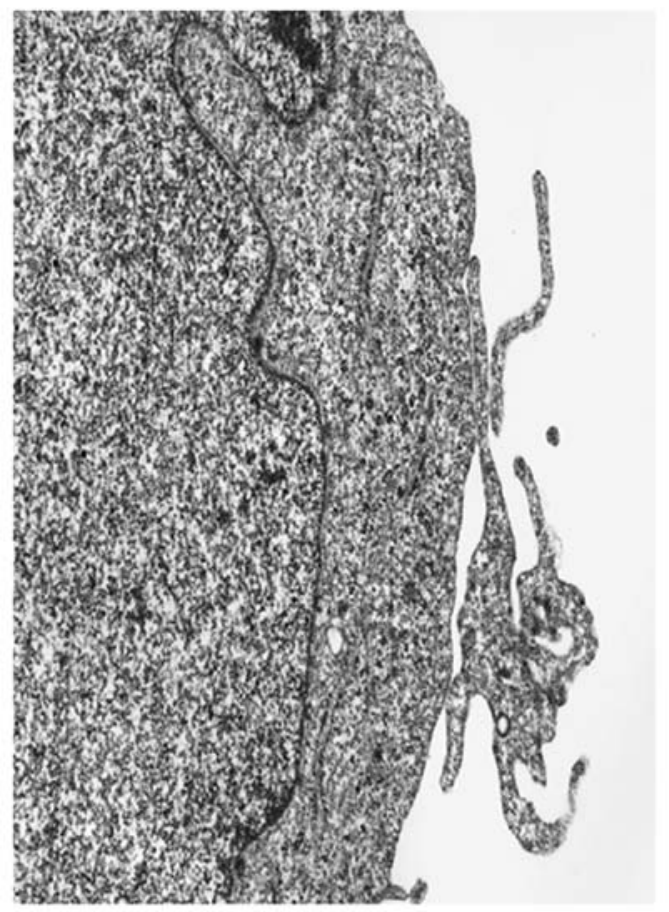

Hand OA

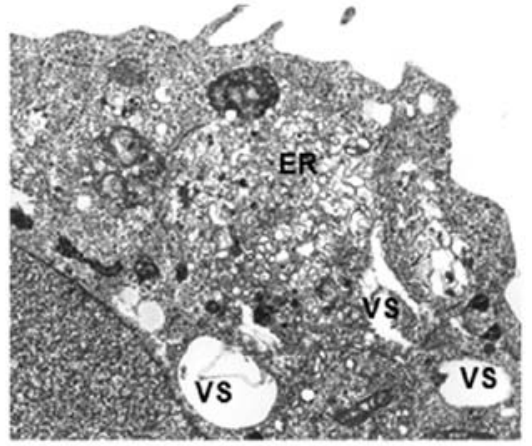

SEMD

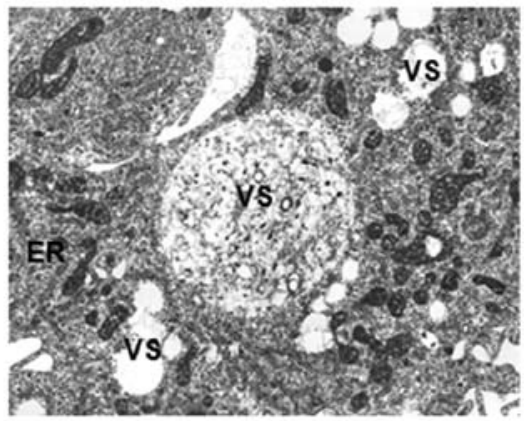

Hand OA + SEMD

Figure 5. Electron microscopy showing an abnormal cytoplasm [expanded endoplasmic reticulum (ER) and multiple vacuoles (VS)] in the cells transfected with the SEMD-associated mutation (C299S) or with SEMD plus HOA-mutant (T298M) matrilin-3 cDNA. Cells were transfected the plasmids carrying the different point mutations [wild-type matrilin-3 or the MED (R116W)-, SEMD (C299S)- and HOA (T298M)-associated mutations, or SEMD plus HOA-mutant matrilin-3 cDNA]. Three days post-transfection, electron microscopy was performed.

A

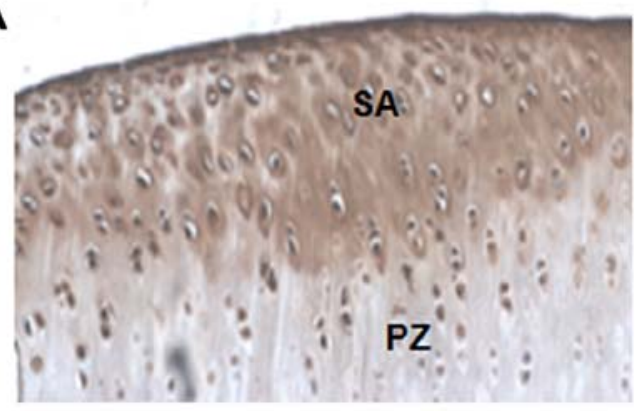

B

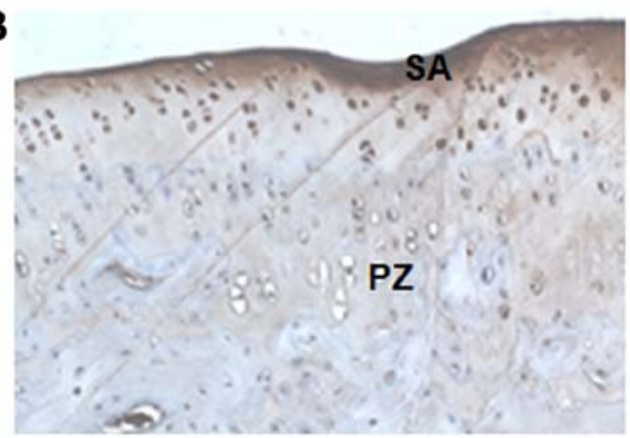

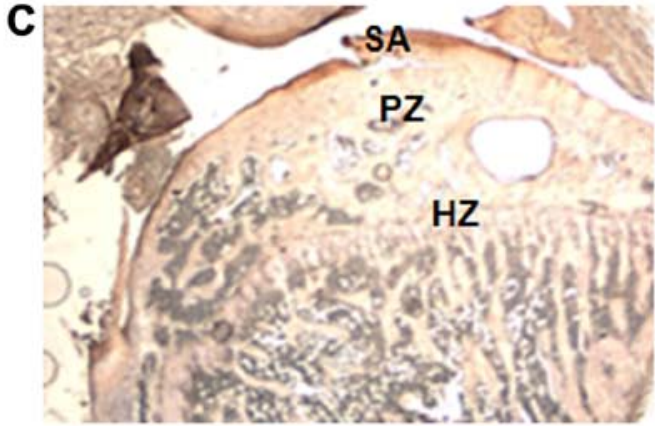

D

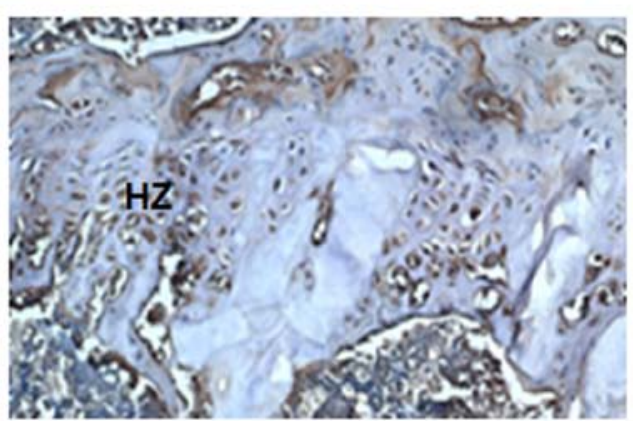

Figure 6. A developmental animal model of osteoarthritis using knee samples obtained from Dunkin-Hartley guinea pigs. This was used to measure matrilin-3 levels in the knee joints by immunohistochemistry. Matrilin-3 was strongly expressed in the articular cartilage, including the surface area (SA), and proliferating (PZ) and hypertrophic areas (HZ). (A) Articular cartilage near the lesion area; (B) articular cartilage in the normal area; (C) overview of tibia of knee joing; (D) hypertrophic areas (HZ).

SEMD (C299S). Our in vitro experiments revealed that there was a decrease in the amount of mutant protein being secreted into the medium of the cells transfected with the plasmid carrying the SEMD-associated mutation (C299S); however, 
Summary of Matrilin-3 Point Mutation

Endoplasmic Reticulum Stress \& Unfolded Protein Response

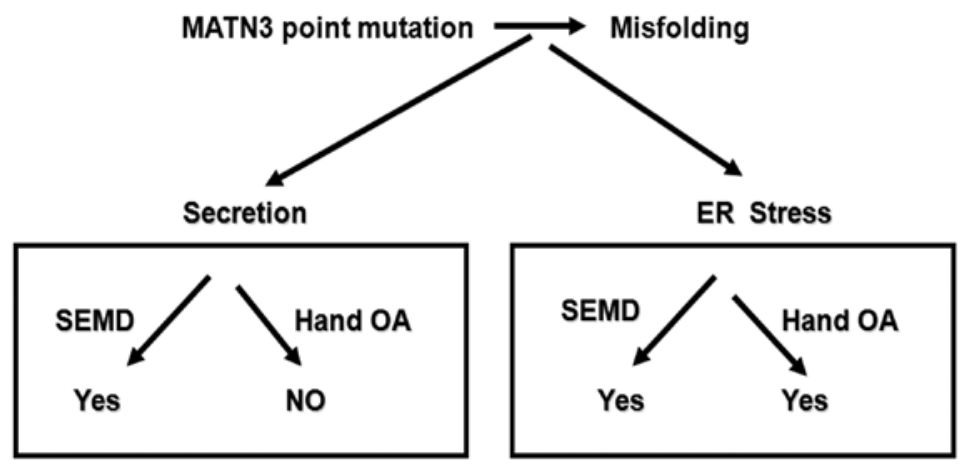

Figure 7. Summary of matrilin-3 point mutations. Different point mutations at the first EGF-like domain in matrilin-3 may lead to distinct pathological mechanisms, due to the multiple functions of this EGF-like domain. Both hand osteoarthritis (HOA) and spondyloepimetaphyseal dysplasia (SEMD) mutations can lead to endoplasmic reticulum (ER) stress and secretion insufficiency; but only SEMD mutation can lead to morphological detectable secretion insufficiency.

this was not observed in the cells transfected with the plasmid carrying the HOA-associated mutation (T298M; secretion was only delayed for $24 \mathrm{~h}$ ) (Fig. 2). In addition, excessive amounts of mutant protein had accumulated intracellularly in the cells transfected with the plasmid carrying the SEMD-associated mutation (Fig. 3). These observations indicate that the intracellular retention of mutant protein is responsible for the deficiency of protein secretion, in terms of both quantity and speed. Consistent with this hypothesis, we observed a marked increase in the number of intracellular vesicles that contained mutant matrilin-3 in the cells transfected with the plasmid carrying the SEMD-associated mutation, as shown by immunofluorescence staining and electron microscopy (Figs. 3 and 5).

The EGF domain is composed of approximately 30-40 amino-acid residues and has been found in a large number of mostly animal proteins; it which is an evolutionary conserved protein domain, which derives its name from EGF, where it was first described $(21,22)$. Most EGF-like domain proteins are found in the extracellular domain of membrane-bound proteins or in proteins known to be secreted. An exception to this is the prostaglandin-endoperoxide synthase. The EGF-like domain includes six cysteine residues which in the epidermal growth factor EGF have been shown to form three disulfide bonds. The structures of 4-disulfide EGF-domains have been solved from the laminin and integrin proteins $(32,33)$. In the present study, although the molecular mechanism of the cysteine mutation of C299S has yet to be investigated, our results suggest that abnormal protein folding contributes to the secretion deficiency of the mutant protein. This abnormal protein folding leads to secretion deficiency, due to intracellular retention of the mutant proteins which may share a common mechanism of matrilin-3-associated MED, COMP-associated MED or related pseudoachondroplasia (34). This retention in turn results in excessive accumulation of the proteins that are associated with COMP, such as collagen type IX, whose mutation also leads to similar clinical manifestations (35). Our observation that cells expressing mutant matrilin-3 exhibit an expanded cytoplasm with multiple vacuoles by immunofluorescence staining and electron microscopy, and our results showing a high level of GADD153 by western blot analysis, which is similar to the phenotype of mutant COMP-expressing cells $(34,35)$, suggests that mutated matrilin-3 or COMP leads to a common cellular phenotype. In light of the discovery that COMP interacts with matrilin-1, -3 and -4 (36), our findings lend support to the hypothesis that mutations in any of these interacting proteins including matrilin, COMP, or collagenIX, result in a secretion defect, which manifests in common chondrodysplasia pathological phenotypes. It should also be noted that a portion of the mutant protein is secreted into the medium. However, we do not know whether the mutant protein is defective in its adhesion to matrix ligands or subject to extracellular proteolysis. These possibilities remain to be determined in future studies.

$\mathrm{OA}$ is one of the most common and disabling diseases in the elderly, affecting nearly $80 \%$ of individuals over the age of 75 (25-27). OA develops due to the wear and tear and the loss of articular cartilage (25-27). It has been previously suggested that cartilage degeneration and insufficient self-repair are the primary cause of OA (25-27). In this study, an developmental animal model of OA with Dunkin-Hartley guinea pigs was used to measure the matrilin-3 expression levels in the knee joints of the guinea pigs by immunohistochemistry. The results revealed that matrilin-3 was strongly expressed in articular cartilage, including the surface area, and proliferating and hypertrophic areas, and its expression level was increased in the articular knee joint cartilage. The results of our examination of the cartilage of the knee joints indicated heterogeneity. A genome-wide linkage analysis of patients with idiopathic HOA who were carefully phenotyped for the involvement of either or both the distal interphalangeal (DIP) joints and the first carpometacarpal (CMC1) joints has previously been performed (24). The missense mutation co-segregates with HOA in several families. The mutation frequency is slightly $>2 \%$ in patients with HOA in the Icelandic population and has a relative risk of 2.1 (24). Previous analysis of recombinant full-length matrilin-3 revealed that the T298M mutation associated with HOA does not influence the oligomerization of matrilin-3 or its proteolytic processing by ADAM metallopeptidase with thrombospondin type 1 motif (ADAMTS)-4 and -5 . Nevertheless, structural analyses indicate local conformational changes. These changes do not affect the affinity for colla- 
gens II, IX, XI or COMP, but have a major impact on the in vitro fibrillogenesis of collagen II/IX/XI heterofibrils (14,28). In the present study, we found the the T298M HOA -associated mutation does not significantly influence protein secretion, but leads to high expression levels of GADD153 (Fig. 4). GADD153, which is a CCAAT/enhancer binding protein $(\mathrm{C} / \mathrm{EBP})$, is a leucine zipper transcription factor that is present in low levels under normal conditions but is robustly expressed in response to stress (29-31). GADD153 was originally identified based on its induction following treatment of cells with growth arresting and DNA-damaging agents, although the induced expression of the gene has also been strongly linked to the perturbation of homeostasis in the ER. It has been proven to be involved in the pathogenesis of various diseases, including brain ischemia (37), Alzheimer's disease (38) and neurodegenerative disease $(37,38)$. The ER stress pathway is activated by GADD153 and is involved in apoptosis. The T298M HOA-associated mutation may be related to fibrillogenesis of collagen II/IX/XI heterofibrils.

In conclusion, the findings of the present study suggest that different point mutations at the first EGF-like domain in matrilin-3 lead to distinct pathological mechanisms due to the multiple functions of this EGF-like domain. This hypothesis would explain how different mutations within matrilin-3 lead to the development of a variety of diseases that affect cartilage (Fig. 7).

\section{Acknowledgements}

The present study was funded partially through the Chinese Natural Grant 81460022 and the New Faculty Foundation of Qinghai Provincial People's Hospital (J.-M.L.) and partially through the Department of Science and Technology of Hunan Province, no. 2013FJ3126, and the Project of Scientific research platform of The Affiliated Cancer Hospital of Xiangya Medical School, Central South University, no. PT2013-09 (Y.-C.W.).

\section{References}

1. Li L, Zhang L, Shao Y, Wang G, Gong R, Wang Z, Peng J, Wang S, Genochio D, Zhao B and Luo J: Distinct roles of two alternative splice variants of matrilin-2 in protein oligomerization and proteolysis. Mol Med Rep 6: 1204-1210, 2012.

2. Krieg T and LeRoy EC: Diseases of the extracellular matrix. J Mol Med Berl 76: 224-225, 1998.

3. Mateos J, De la Fuente A, Lesende-Rodriguez I, FernándezPernas P, Arufe MC and Blanco FJ: Lamin A deregulation in human mesenchymal stem cells promotes an impairment in their chondrogenic potential and imbalance in their response to oxidative stress. Stem Cell Res (Amst) 11: 1137-1148, 2013.

4. Ankam S, Teo BK, Kukumberg M and Yim EK: High throughput screening to investigate the interaction of stem cells with their extracellular microenvironment. Organogenesis 9: 128-142, 2013.

5. Chen Q, Zhang Y, Johnson DM and Goetinck PF: Assembly of a novel cartilage matrix protein filamentous network: molecular basis of differential requirement of von Willebrand factor A domains. Mol Biol Cell 10: 2149-2162, 1999.

6. Frank S, Schulthess T, Landwehr R, Lustig A, Mini T, Jenö P, Engel $\mathrm{J}$ and Kammerer RA: Characterization of the matrilin coiled-coil domains reveals seven novel isoforms. J Biol Chem 277: 19071-19079, 2002.

7. Klatt AR, Becker AK, Neacsu CD, Paulsson M and Wagener R: The matrilins: modulators of extracellular matrix assembly. Int J Biochem Cell Biol 43: 320-330, 2011.

8. Paulsson M, Piecha D, Segat D, Smyth N and Wagener R: The matrilins: a growing family of A-domain-containing proteins. Biochem Soc Trans 27: 824-826, 1999.
9. Piecha D, Muratoglu S, Mörgelin M, Hauser N, Studer D, Kiss I, Paulsson M and Deák F: Matrilin-2, a large, oligomeric matrix protein, is expressed by a great variety of cells and forms fibrillar networks. J Biol Chem 274: 13353-13361, 1999.

10. Whittaker CA and Hynes RO: Distribution and evolution of von Willebrand/integrin A domains: widely dispersed domains with roles in cell adhesion and elsewhere. Mol Biol Cell 13: 3369-3387, 2002.

11. Luo $\mathbf{J}$ and Wan Y: Tightly regulated distribution of family members of proteins is related to social property in the open body system (Review). Int J Mol Med 17: 411-418, 2006.

12. Belluoccio D, Schenker T, Baici A and Trueb B: Characterization of human matrilin-3 (MATN3). Genomics 53: 391-394, 1998.

13. Zhang $\mathrm{Y}$ and Chen Q: Changes of matrilin forms during endochondral ossification. Molecular basis of oligomeric assembly. J Biol Chem 275: 32628-32634, 2000.

14. van der Weyden L, Wei L, Luo J, Yang X, Birk DE, Adams DJ, Bradley A and Chen Q: Functional knockout of the matrilin-3 gene causes premature chondrocyte maturation to hypertrophy and increases bone mineral density and osteoarthritis. Am J Pathol 169: 515-527, 2006.

15. Zhang Y, Wang ZK, Luo JM, Kanbe K and Chen Q: Multiple functions of the von Willebrand Factor A domain in matrilins: secretion, assembly, and proteolysis. J Orthop Surg 3: 21, 2008.

16. Chapman KL, Mortier GR, Chapman K, Loughlin J, Grant ME and Briggs MD: Mutations in the region encoding the von Willebrand factor A domain of matrilin-3 are associated with multiple epiphyseal dysplasia. Nat Genet 28: 393-396, 2001.

17. Jackson GC, Barker FS, Jakkula E, Czarny-Ratajczak M, Mäkitie O, Cole WG, Wright MJ, Smithson SF, Suri M, Rogala $\mathrm{P}$, et al: Missense mutations in the beta strands of the single A-domain of matrilin-3 result in multiple epiphyseal dysplasia. J Med Genet 41: 52-59, 2004.

18. Mäkitie O, Mortier GR, Czarny-Ratajczak M, Wright MJ, Suri M, Rogala P, Freund M, Jackson GC, Jakkula E, Ala-Kokko L, et al: Clinical and radiographic findings in multiple epiphyseal dysplasia caused by MATN3 mutations: description of 12 patients. Am J Med Genet A 125A: 278-284, 2004.

19. Mabuchi A, Haga N, Maeda K, Nakashima E, Manabe N, Hiraoka $\mathrm{H}$, Kitoh H, Kosaki R, Nishimura G, Ohashi H and Ikegawa S: Novel and recurrent mutations clustered in the von Willebrand factor A domain of MATN3 in multiple epiphyseal dysplasia. Hum Mutat 24: 439-440, 2004.

20. Cotterill SL, Jackson GC, Leighton MP, Wagener R, Mäkitie O, Cole WG and Briggs MD: Multiple epiphyseal dysplasia mutations in MATN3 cause misfolding of the A-domain and prevent secretion of mutant matrilin-3. Hum Mutat 26: 557-565, 2005.

21. Wouters MA, Rigoutsos I, Chu CK, Feng LL, Sparrow DB and Dunwoodie SL: Evolution of distinct EGF domains with specific functions. Protein Sci 14: 1091-1103, 2005.

22. Rao Z, Handford P, Mayhew M, Knott V, Brownlee GG and Stuart D: The structure of a $\mathrm{Ca}^{2+}$-binding epidermal growth factor-like domain: its role in protein-protein interactions. Cell 82: 131-141, 1995.

23. Borochowitz ZU, Scheffer D, Adir V, Dagoneau N, Munnich A and Cormier-Daire V: Spondylo-epi-metaphyseal dysplasia (SEMD) matrilin 3 type: homozygote matrilin 3 mutation in a novel form of SEMD. J Med Genet 41: 366-372, 2004

24. Stefánsson SE, Jónsson H, Ingvarsson T, Manolescu I, Jónsson HH, Olafsdóttir G, Pálsdóttir E, Stefánsdóttir G, Sveinbjörnsdóttir G, Frigge ML, et al: Genomewide scan for hand osteoarthritis: a novel mutation in matrilin-3. Am J Hum Genet 72: 1448-1459, 2003.

25. Zhang S, Peng J, Guo Y, Javidiparsijani S, Wang G, Wang Y, Liu H, Liu J and Luo J: Matrilin-2 is a widely distributed extracellular matrix protein and a potential biomarker in the early stage of osteoarthritis in articular cartilage. Biomed Res Int 2014: 986127, 2014.

26. WeiF, Moore DC, Wei L,Li Y,Zhang G, Wei X,Lee JK and Chen Q: Attenuation of osteoarthritis via blockade of the SDF-1/CXCR4 signaling pathway. Arthritis Res Ther 14: R177, 2012.

27. Lawrence RC, Felson DT, Helmick CG, Arnold LM, Choi H, Deyo RA, Gabriel S, Hirsch R, Hochberg MC, Hunder GG, et al; National Arthritis Data Workgroup: Estimates of the prevalence of arthritis and other rheumatic conditions in the United States. Part II. Arthritis Rheum 58: 26-35, 2008.

28. Otten C, Wagener R, Paulsson M and Zaucke F: Matrilin-3 mutations that cause chondrodysplasias interfere with protein trafficking while a mutation associated with hand osteoarthritis does not. J Med Genet 42: 774-779, 2005. 
29. Liu H, Qian J, Wang F, Sun X, Xu X, Xu W, Zhang X and Zhang X: Expression of two endoplasmic reticulum stress markers, GRP78 and GADD153, in rat retinal detachment model and its implication. Eye (Lond) 24: 137-144, 2010.

30. Feng LJ, Jiang TC, Zhou CY, Yu CL, Shen YJ, Li J and Shen YX: Activated macrophage-like synoviocytes are resistant to endoplasmic reticulum stress-induced apoptosis in antigen-induced arthritis. Inflamm Res 63: 335-346, 2014.

31. Carlisle RE, Brimble E, Werner KE, Cruz GL, Ask K, Ingram AJ and Dickhout JG: 4-Phenylbutyrate inhibits tunicamycininduced acute kidney injury via CHOP/GADD153 repression. PLoS One 9: e84663, 2014.

32. Downing AK, Knott V, Werner JM, Cardy CM, Campbell ID and Handford PA: Solution structure of a pair of calcium-binding epidermal growth factor-like domains: implications for the Marfan syndrome and other genetic disorders. Cell 85: 597-605, 1996.

33. Bork P, Downing AK, Kieffer B and Campbell ID: Structure and distribution of modules in extracellular proteins. Q Rev Biophys 29: 119-167, 1996.
34. Vranka J, Mokashi A, Keene DR, Tufa S, Corson G, Sussman M, Horton WA, Maddox K, Sakai L and Bächinger HP: Selective intracellular retention of extracellular matrix proteins and chaperones associated with pseudoachondroplasia. Matrix Biol 20: 439-450, 2001.

35. Hecht JT, Makitie O, Hayes E, Haynes R, Susic M, Montufar-Solis D, Duke PJ and Cole WG: Chondrocyte cell death and intracellular distribution of COMP and type IX collagen in the pseudoachondroplasia growth plate. J Orthop Res 22: 759-767, 2004.

36. Mann HH, Ozbek S, Engel J, Paulsson M and Wagener R: Interactions between the cartilage oligomeric matrix protein and matrilins. Implications for matrix assembly and the pathogenesis of chondrodysplasias. J Biol Chem 279: 25294-25298, 2004.

37. Oyadomari S and Mori M: Roles of CHOP/GADD153 in endoplasmic reticulum stress. Cell Death Differ 11: 381-389, 2004.

38. Prasanthi JRP, Larson T, Schommer J and Ghribi O: Silencing GADD153/CHOP gene expression protects against Alzheimer's disease-like pathology induced by 27 -hydroxycholesterol in rabbit hippocampus. PLoS One 6: e26420, 2011. 